Results A total of 40,217 women were included in the analyses, of whom $15.4 \%$ tested chlamydia positive genitally at their second test. Not being tested anorectally at the first test was an independent risk factor of genital chlamydia infection at the repeat test (aOR 1.24 [95\%CI 1.15-1.33]). This effect was in the same range as most other significant risk factors in the model: low education level, no condom use, STI symptoms and previous STI diagnosis. Young age $(<20$ years 2.67, [2.39-2.98]) and received partner notification (3.11, [2.913.31]) showed stronger effects. The findings were robust: correcting for interactions and a sensitivity analysis stratifying by chlamydia infection at first visit did not show significant differences in the aOR of not being tested anorectally at first test.

Conclusion The results are suggestive of an autoinoculation process from the anorectal to the genital anatomical site in women. To enhance chlamydia control, future studies on the role of extragenital testing and autoinoculation in chlamydia transmission are needed.

\section{P108 PREVALENCE AND EPIDEMIOLOGICAL FACTORS ASSOCIATED WITH TRICHOMONAS VAGINALIS INFECTION IN A US MULTICENTER STI CLINICAL STUDY}

J Kostera*. Abbott Laboratories, Des Plaines, USA

10.1136/sextrans-2021-sti.233

Trichomonas vaginalis (TV) is the most common, curable nonviral sexually transmitted infection (STI) in the world, yet the epidemiology of the infection in the United States is not well defined. The infection is often associated with preterm birth, low-birthweight infants, infertility, as well as a facilitator for increasing the risk for acquisition and transmission of human immunodeficiency virus (HIV). Risk factors for TV in women include older age, race, impoverished socioeconomic status, and multiple sexual partners. The epidemiology of TV in men is less delineated, as men are not routinely tested, and most infections are asymptomatic.

In this work, we present TV prevalence in conjunction with Mycoplasma genitalium, Chlamydia trachomatis, and Neisseria gonorrhoeae prevalence with socio-demographic epidemiological data associated with patients enrolled in a multicenter STI study. Prevalence data was generated using the automated multiplex qualitative CE marked assay, Alinity m STI. The study included symptomatic and asymptomatic patients including a cohort of individuals who have been previously diagnosed with an STI seeking treatment at public health clinics and primary care offices within the United States.

The prevalence of TV in women was determined to be $12.3 \%$ and $0.6 \%$ in men. When stratified by ethnicity and prior STI history in this cohort, the positivity rate of TV in African American women was $22.3 \%$ compared to $1.6 \%$ in White women. Furthermore, in this data set, African American women 40 and older had the highest TV positivity rate at $26.5 \%$ compared to $22.9 \%$ for those $30-39$, and $21.1 \%$ for those less than 29 years old. Those who reported not being diagnosed with a prior STI had a TV prevalence of $16.7 \%$ for African American women compared to $1.3 \%$ of White women. In this study, the overall prevalence of Chlamydia trachomatis was 9.8\%, Neisseria gonorrhoeae was $2.4 \%$, and Mycoplasma genitalium was $11.6 \%$.

\section{P112 REMOVING BARRIERS TO HIV SCREENING IN A COMMUNITY-BASED PEDIATRIC EMERGENCY DEPARTMENT}

${ }^{1} \mathrm{M}$ Gaines*, '1J Wiggins, 'B Sierzant, ${ }^{2,3}$ B Coleman, ${ }^{1,2} \mathrm{~W}$ Hannah. ${ }^{1}$ Memorial Health University Medical Center, Savannah, USA; ${ }^{2}$ Mercer University School of Medicine, Savannah, USA; ${ }^{3}$ Georgia Emergency Physicians Specialists, Savannah, USA

\subsection{6/sextrans-2021-sti.234}

Background Memorial University Medical Center conducts opt-out HIV screening in the Emergency Department (ED) for patients aged $\geq 13$. After gaining consent, an electronic algorithm automatically orders an HIV test with the diagnostic evaluation for patients who meet CDC guidelines for HIV screening. Unfortunately, no pediatric patient aged 13-18 received HIV screening despite our out-opt testing protocol being in place since 2016 .

Approach Through a systematic analysis, implicit bias to gain consent was repeatedly identified as the most common barrier to HIV screening in this population. Pediatric leadership developed strategies to improve HIV screening, including education of parents and staff, on-site support for staff and parents/ guardians, pediatric patient counseling, and adding HIV screening to syphilis tests. Despite these interventions, HIV screening in the pediatric population only improved about $37 \%$ from January 2017 through November 2020. As a final strategy HIV screening was added to the sexually transmitted infection (STI) order set for patients who met CDC guidelines for STI. Pediatric ED HIV education to clinical staff continued, specifically emphasizing the high prevalence of HIV in our community.

Outcomes Persistence to drive change increased HIV screening by $61 \%$ from July 2020 to December 2020. Strategies producing the greatest impact were the inclusion of HIV testing in the standard STI panel and focused pediatric ED nurse education/support. As a result, current HIV screening in our pediatric patients aged $13-18$ is now $88 \%$ of those who meet CDC guidelines to be screened.

Significance Up to $20 \%$ of HIV diagnoses occur during adolescence making screening in this age group imperative. Removing implicit bias from HIV screening is difficult. However, continued persistence and automated testing protocols can lead to increases in pediatric ED HIV screening. Because of our high HIV screening rate, many pediatric patients will have early identification of HIV and linkage to care.

\section{P113 ACCEPTABILITY AND PREFERENCES FOR USING A NOVEL DEVICE TO SELF-COLLECT BLOOD SPECIMENS FOR HIV PRE-EXPOSURE PROPHYLAXIS (PREP) LABORATORY MONITORING}

${ }^{1,2} \mathrm{C}$ Cannon*, ${ }^{1,2} \mathrm{C}$ Malinski, ${ }^{1,2} \mathrm{M}$ Golden. 'University of Washington, Seattle, USA; ${ }^{2}$ Public Health-Seattle and King County, Seattle, USA

10.1136/sextrans-2021-sti.235

Home-based PrEP monitoring (HBPM) has the potential to improve PrEP access and retention but is hampered by difficulties collecting blood, which typically involves painful fingersticks that yield insufficient specimen for quantitative syphilis serologies. We evaluated the use of the Tasso OnDemand ${ }^{\mathrm{TM}}$, a device that facilitates relatively painless self-collection of 